\title{
Emergence of Particle-Hole Symmetry near Optimal Doping in High-Temperature Copper Oxide Superconductors
}

\author{
Shiladitya Chakraborty, Dimitrios Galanakis, and Philip Phillips \\ Department of Physics, University of Illinois 1110 W. Green Street, Urbana, IL 61801, U.S.A.
}

(Dated: November 17, 2018)

\begin{abstract}
High-temperature copper oxide superconductors (cuprates) display unconventional physics when they are lightly doped whereas the standard theory of metals prevails in the opposite regime. For example, the thermoelectric power, that is the voltage that develops across a sample in response to a temperature gradient, changes sign abruptly near optimal doping in a wide class of cuprates, a stark departure from the standard theory of metals in which the thermopower vanishes only when one electron exists per site. We show that this effect arises from proximity to a state in which particle-hole symmetry is dynamically generated. The operative mechanism is dynamical spectral weight transfer from states that lie at least $2 \mathrm{eV}$ away from the chemical potential. We show that the sign change is reproduced quantitatively within the Hubbard model for moderate values of the on-site repulsion, $U$. For sufficiently large values of on-site repulsion, for example, $U=20 t,(t$ the hopping matrix element), dynamical spectral weight transfer attenuates and our calculated results for the thermopower are in prefect agreement with exact atomic limit. The emergent particle-hole symmetry close to optimal doping points to pairing in the cuprates being driven by high-energy electronic states.
\end{abstract}

\section{INTRODUCTION}

As charge carriers are doped into the wide family of parent copper-oxide ceramics (cuprates), superconductivity obtains with a maximum transition temperature at a particular chemical composition. The result is a dome-shaped superconducting region in the dopingtemperature plane. The origin of the dome remains pivotal to the solution of this problem because the properties of all high $T_{c}$ superconductors change drastically around optimal doping, $x_{c}$. Unconventional physics pervades for $x<x_{c}$ in which the standard theory of metals breaks down whereas such a description is recovered in the opposite regime. The efficient cause underlying this drastic change in the physics of the cuprates as the dome is traversed remains one of the key mysteries of these materials. To solve this problem, it is instructive to focus on a correlate of superconductivity, that is a phenomenon which supervenes on superconductivity and exhibits an abrupt signature at the top of the dome. Such a correlate of superconductivity could elucidate the ubiquitous dome-like structure in the doping-temperature plane of the cuprates.

To this end, we consider the thermo-electric power $(S)$, defined as the voltage difference that develops across a material in response to a temperature gradient. Physically, the thermopower measures the entropy per carrier and is a direct probe of particle number rather than charge conservation, a distinction which is of utmost importance in this work. A universal feature observed amongst different families of hole-doped cuprates ${ }^{1-5}$ is that the thermo-electric power is non-zero everywhere except at one particular doping value. Shown in Fig. (19) is a collation of thermopower data at $290 \mathrm{~K}$ for a wide range of hole-doped cuprates as a function of the planar hole density per unit area, $P_{\mathrm{pl}}$, which measures the hole concentration in each $\mathrm{CuO}_{2}$ layer. $P_{\mathrm{pl}}$ provides an even-handed way of comparing different families of cuprates that is independent of the doping method or sample quality, be they single crystals or not. As long as $x$ refers to the number of holes per $\mathrm{CuO}_{2}$ layer, $x$ and $P_{\mathrm{pl}}$ can be used interchangeably as we do here. Clearly shown is that for all hole-doped cuprates, the thermopower at $290 \mathrm{~K}$ is positive for $P_{\mathrm{pl}}<0.24$, whereas for $P_{\mathrm{pl}}>0.24$, it is negative. To correlate this sign change with superconductivity, Honma and Hor ${ }^{1}$ used the thermopower doping scale as opposed to the Presland scale $e^{6}, 1-T_{c} / T_{c}^{\max }=82.6(x-0.16)^{2}$, in which the maximum $T_{c}$ is artificially fixed to be 0.16 , to determine the doping level at which $T_{c}$ is maximized. We show in Fig. (1) the maximum superconducting transition temperature $T_{c}^{\max }$ as a function of $P_{\mathrm{pl}}$ for 23 different types of cuprates classified by the number of copper-oxide layers in each unit cell. Of the 23 cuprates shown, only four single-layer materials exhibit any significant deviation of the maximum $T_{c}$ from the planar hole density at which the thermopower vanishes.

One might argue that this agreement is irrelevant since the thermopower is highly temperature dependent. However, experiments ${ }^{7}$ indicate that at $110 \mathrm{~K}$, the zero-crossing of the thermopower is shifted to 0.26 , quite similar to the value at $290 \mathrm{~K}$. In addition, one might also question the validity of the thermopower scale to calibrate the doping level. To address this question, we reprint here a figure (see Fig. (21) from their paper which illustrates that the thermopower scale is in excellent agreement with the doping level in Y123 determined by three different experimental methods. Hence, the zero-crossing in the vicinity of $T_{c}^{\max }$ is a robust feature of the cuprates that has direct bearing on why $T_{c}$ is 
(a)

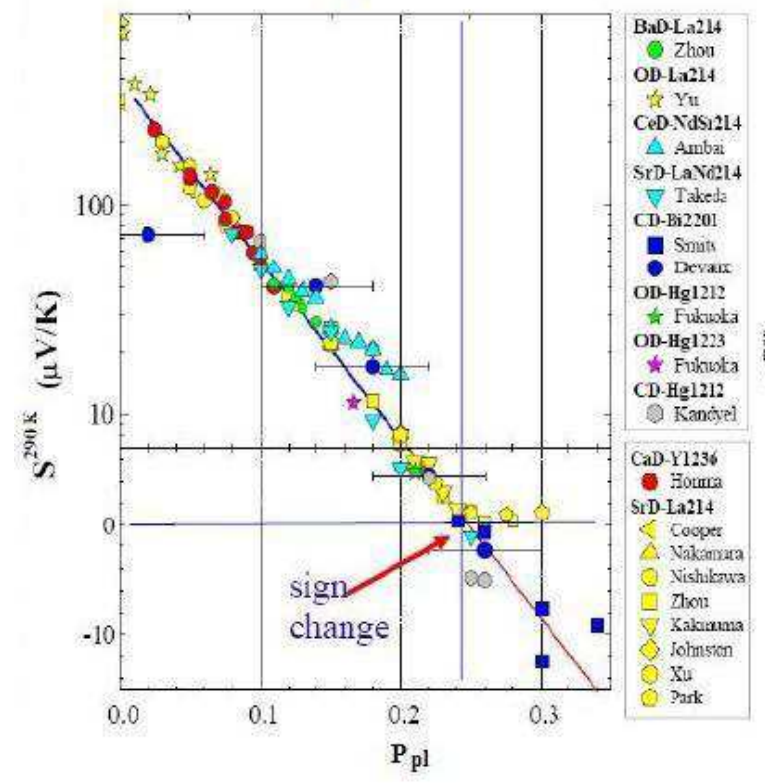

(b)

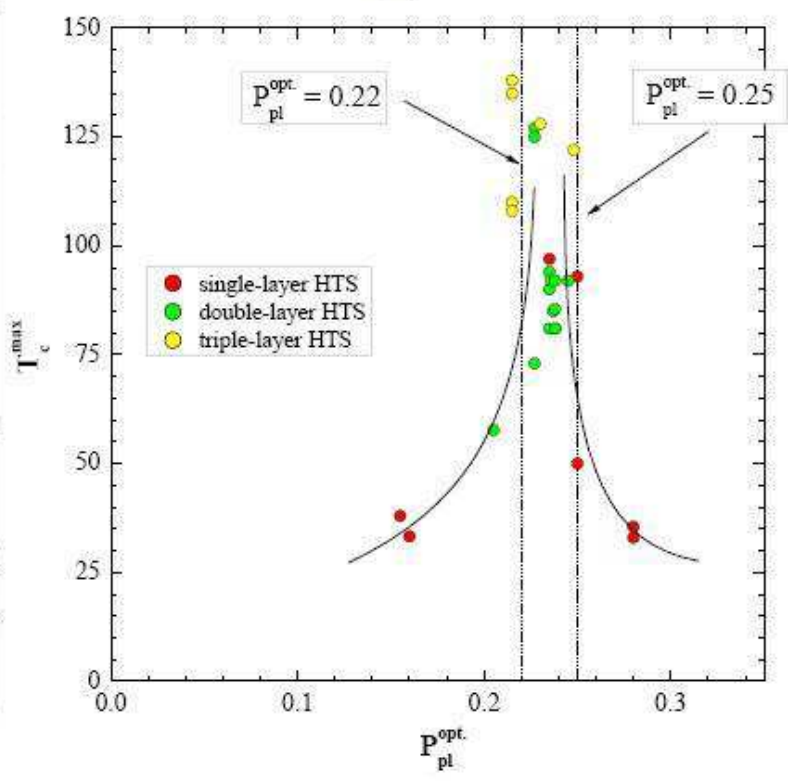

FIG. 1. a) Universal behavior of the thermoelectric power ${ }^{1}$ $(290 \mathrm{~K})$ as a function of planar hole density $\left(P_{\mathrm{pl}}\right)$, for various families of hole-doped cuprates. All exhibit a sign change at $P_{\mathrm{pl}}=0.23$. Above the solid-bold horizontal line, the thermpower obeys the functional form, $S^{290}\left(P_{\mathrm{pl}}\right)=$ $392 \exp \left(-19.7 P_{\mathrm{pl}}\right)$ for $0.01<P_{\mathrm{pl}}<0.21$. Below the solid-bold horizontal line, $S^{290}\left(P_{p l}\right)=40.47-163.4 P_{\mathrm{pl}}$ for $0.21<P_{\mathrm{pl}}<$ 0.34. These functional forms were used ${ }^{1}$ to determine the hole doping levels for all the cuprates rather than the widely used empirical formula ${ }^{6} 1-T_{c} / T_{c}^{\max }=82.6(x-0.16)^{2}$ which artificially fixes the optimal doping level of all cuprates to be 0.16. b) Maximum transition temperature as a function of the planar hole density using the thermopower scale to determine the doping level. Except for three single-layer materials, the vanishing of the thermopower coincides with the doping level at which the transition temperature is maximized.

so high. Our analysis indicates that mixing with states that lie at least $2 \mathrm{eV}$ (the optical gap in the cuprates $\underline{\underline{8}} \underline{\underline{11}}, 13,14$ ) away from the chemical potential acount for the dramatic sign change of the thermopower and hence are the cause of the high transition temperature for most of the cuprates, particularly those exhibiting $T_{c}>70 \mathrm{~K}$ (see Fig. (1) ).

\section{THERMOPOWER AND SPECTRAL WEIGHT TRANSFER}

The dramatic sign change of the thermopower in the cuprates poses a distinct theoretical problem because it represents a stark departure from the predictions of freeelectron physics. For example, in the standard theory of metals, Fermi liquid theory, the sign of the thermopower unambiguously reveals the sign of the dominant charge carriers in the material. Consequently, in the standard theory of metals, the thermopower only vanishes in a half-filled band where the number of filled and empty states is equal, the particle-hole symmetric condition. In the cuprates, the parent materials possess a half-filled band. Doping away from half-filling by the introduction of holes should only increase the particle-hole asymmetry. Consequently, it is unexpected that the thermopower should vanish at $x=0.24$ corresponding to a filling of $n=0.76$. Existing treatments of the thermopower in the cuprates address either the low-doping regime ${ }^{21}$ or invoke special band structure effects such as Van Hove singularities ${ }^{22}$ and the shape of the Fermi surface ${ }^{23}$ to describe the sign change. However, given that the sign change obtains near optimal doping, the approximate crossover from strong to weakly interacting physics, the explanation of the sign change of $S$, must incorporate the 


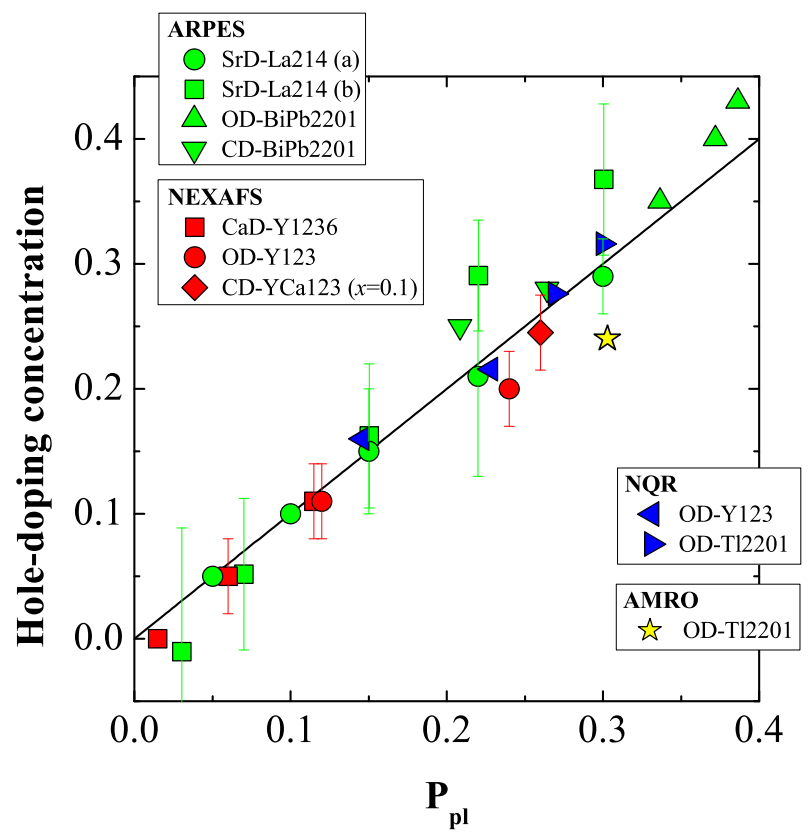

FIG. 2. Hole-doping level from various techniques compared with the doping scale extracted from the thermopower, $P_{\mathrm{pl}}$. The red points are obtained from (near-edge X-ray absorption fine structure) NEXAFS: red circles are OD-Y123 ${ }^{15}$, red diamonds are co-doped Ca-YaC123 (at $\mathrm{x}=0.1)^{15}$, red squares are Calcium-doped Y1236 $\frac{15}{5}$. The blue points are from nuclear-quadrupole resonance (NQR) measurements: left blue arrow is OD-Y $123^{16}$ and the right blue arrow is OD-Ti2201 ${ }^{16}$. The green points are from ARPES: green circles are Strontium-doped La214 ${ }^{17}$, green squares are also Strontium-doped La214 ${ }^{18}$, uprrow, overdoped $\mathrm{BiPb} 2201^{19}$, down arrow co-doped BiP2201 ${ }^{19}$. The star corresponds to angular magnetoresistance oscillations $(\mathrm{AMRO})^{20}$. Reprinted from Phys. Rev. B 77, 84520 (2008).

physics of strong correlations.

To address this question, we focus on the minimal model that captures the strong correlations of the copperoxide planes of the cuprates. While what constitutes the minimal model for the cuprates can certainly be debated, it is clear ${ }^{24-27}$ that regardless of the model, the largest energy scale arises from doubly occupying the copper $d_{x^{2}-y^{2}}$ orbital. This orbital can hybridise with the in-plane $p_{x}$ and $p_{y}$ orbitals and hence a three-band model is natural. As emphasized earlier ${ }^{25}$, the hybridization with the d-orbitals in the cuprates is sizeable. In so far as spectral weight transfer is concerned 25 , the 3 -band model with the p-d hybridization that is relevant for the cuprates and the one-band Hubbard models are essentially identical for hole doping. As our primary emphasis here is spectral weight transfer, we adopt the one-band Hubbard model,

$$
H_{\mathrm{Hubb}}=-t \sum_{i, j, \sigma} g_{i j} c_{i, \sigma}^{\dagger} c_{j, \sigma}+U \sum_{i, \sigma} c_{i, \uparrow}^{\dagger} c_{i, \downarrow}^{\dagger} c_{i, \downarrow} c_{i, \uparrow},
$$

where $i, j$ label lattice sites, $g_{i j}$ is equal to one iff $i, j$ are nearest neighbours, $c_{i \sigma}$ annihilates an electron with spin $\sigma$ on lattice site $i, t$ is the nearest-neighbour hopping matrix element and $U$ the energy cost when two electrons doubly occupy the same site. Nonetheless, our conclusions carry over naturally to any n-band model of the cuprates as long as the largest energy scale is the onsite energy, $U$ in Eq. (11). For the cuprates $U \approx 4.0 \mathrm{eV}$ and $t \approx 0.5 \mathrm{eV}$. Consequently, the cuprates reside in the strong-coupling regime where standard weak perturbative treatments breakdown.

Quite generally, the strong correlations in the Hubbard model mediate a universal sign change in the thermopower at non-integer fillings as seen in the cuprates. Since $U \gg t$ in the cuprates, perturbation around the atomic limit (vanishing hopping, $t=0$ ) rather than the non-interacting regime $(U=0)$ is the correct starting point. In 1974, Beni28 considered this limit of the Hubbard model and calculated the exact expression for the thermopower

$$
S=-\frac{k_{B}}{e} \ln \frac{2 x}{1-x}
$$

to $O(t / U)$ and $U \gg k_{B} T$, where $k_{B}$ is Boltzmann's constant and $e$ is the electric charge. This expression vanishes exactly at a hole doping level of $x=1 / 3$. However, the reason why it vanishes at $x=1 / 3$ has never been understood. Consider the general expression ${ }^{29,30}$ for the 
thermopower ,

$$
S=-\frac{k_{B}}{e} \beta \frac{L_{12}}{L_{11}},
$$

with the transport integrals $L_{i j}$ in the relaxation-time approximation given by

$$
L_{i j}=\int_{-\infty}^{\infty} d \omega\left(-\frac{\partial f(\omega)}{\partial \omega}\right) \tau^{i}(\omega) \omega^{j-1}
$$

and $\tau(\omega)$ being the relaxation time,

$$
\tau(\omega)=\frac{1}{N} \sum_{\mathbf{k}, \sigma}\left(\frac{\partial \epsilon_{\mathbf{k}}}{\partial k_{x}}\right)^{2} A^{2}(\mathbf{k}, \omega)
$$

In the atomic limit, the single-particle spectral function, $A(k, \omega)$, which when summed over momentum yields the density of states, has no momentum dependence. We have used natural units in which $\hbar=m_{e}=1$. Consequently, from the form of $L_{12}$, a vanishing of the thermopower arises entirely particle-hole symmetry. How does such a particle-hole symmetry obtain for $x=1 / 3$ in a Hubbard system? Alternatively, what is so special about the $(2 x / 1-x)$ ratio in the argument of the logarithm in Eq. (2)? The answer lies in spectral weight transfer which was only understood as the signature physical phenomenon ${ }^{31,33,35}$ of strongly correlated systems in the early 1990's, roughly 20 years after Eq. (2) was derived. As no one yet has presented an explanation of the Beni28 result in this light, we offer one here. In the atomic limit of the Hubbard model, as depicted in Fig. (3), one particle resides per site. In a Hubbard system consisting of $N$ sites, there are $N$ ways to remove an electron and $N$ ways to add one, constituting the photoemission and inverse photoemission bands, respectively. In the atomic limit, the splitting between these bands (also known as the lower and upper Hubbard bands) is the Mott gap, $U$, as shown clearly in Fig. (3). Now consider removing a particle. There are now $N-1$ ways to remove a particle and $N+1$ ways to add a particle. However, only $N-1$ such states lie in the high energy scale as there are only $N-1$ ways to add a particle to the system so that it costs an energy $U$. The two remaining states correspond to the two ways of adding an electron with either spin up or spin down to the empty site. Such addition processes cost no energy and hence must lie directly above the chemical potential. In general if $x$ holes are introduced, the spectral weight immediately above the chemical potential grows as $2 x$ whereas the weight in the lower band decreases as $1-x$. The ratio in the Beni 28 expression is simply the number of states above and below the chemical potential in the photoemission band in the atomic limit. When the two spectral weights are equal, particle-hole symmetry obtains and the thermopower vanishes. The exact particle-hole symmetry condition for the photoemission band of a Mott insulator is $2 x=1-x$. As a result, in a strongly-correlated system, spectral weight transfer and the thermopower are intimately linked.

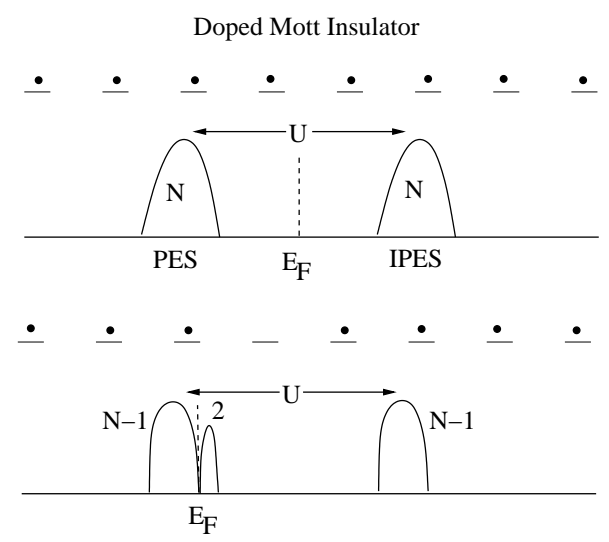

FIG. 3. Evolution of the single-particle density of states from half-filling to the one-hole limit in a doped Mott insulator described by the Hubbard model. Removal of an electron results in two empty states at low energy as opposed to one in the band-insulator limit. The key difference with the Fermi liquid is that the total weight spectral weight carried by the lower Hubbard band (analogue of the valence band in a Fermi liquid) is not a constant but a function of the filling.

Real Mott systems are not in the atomic limit because $t / U \neq 0$. This results in two new effects, the second of which destroys the necessity for a strict particle-hole symmetry to be present for the thermopower to vanish. First, the number of particle addition states immediately above the chemical potential,

$$
L=\int_{\mu}^{\Lambda} N(\omega) d \omega
$$

computed by integrating the single-particle density of states from the chemical potential to a cut-off energy that demarcates the low from the high energy scale, exceeds $2 x^{\underline{32}}$. The reason is simple. Away from the atomic limit, no eigenstate of the Hubbard model involves a fixed number of doubly occupied sites. Harris and Lange 32 that such mixing with the high-energy scale leads to an increase in the weight of the lower band to $1+x+\alpha$, where $\alpha$ represents all the correction due to the mixing with the upper band. $\alpha$ is strictly positive as shown by Harris and Lange ${ }^{32}$ and confirmed by numerics 31 . Because the total weight of both bands is 2 , the weight of the upper band is $1-x-\alpha$. In general $\alpha$ is doping dependent and can be as large as $20 \% \underline{\underline{34}}$. To interpret the thermopower in terms of the entropy, it is helpful to reinstate an atomic-limit picture of the LHB. We have shown ${ }^{34}$ how this can be done by re-defining doping level to include the holes that are dynamically generated as a result of mixing with the upper Hubbard band. To this end, we define $x^{\prime}=x+\alpha$. Consequently, the filled part of the spectrum at low energies has a weight of $1-x^{\prime}$ and the empty part $2 x^{\prime}$. Hence, $L=2 x^{\prime}>2 x$ as a result of dynamical spectral weight transfer. The new particle-hole symmetric condition for the lower band is $1-x-\alpha=2(x+\alpha)$ or $x_{\mathrm{phs}}=1 / 3-\alpha$ which is strictly less than $1 / 3$, the atomic-limit value. 
However, a strict particle-hole symmetry is not necessary for the thermopower to vanish once the hopping is turned on. At play here is the fact that the spectral function is momentum dependent for $t \neq 0$. As a result, the vanishing of $L_{12}$ no longer arises from a simple balancing between the density of states above and below the chemical potential, but rather $\tau(\omega)$ averaged above and below the chemical potential must be equal. This pushes the zero crossing of the thermopower to even lower doping levels. We note importantly that computational techniques will differ in the spectral function which in turn will affect the magnitude of the thermopower. However, the existence of the zero crossing and its location relative to $x<1 / 3-\alpha$ is a robust feature determined entirely by the integrated weights of the spectral intensity below and above the chemical potential and not on the computational method used.

\section{REALISTIC CALCULATIONS}

To quantify the arguments presented here, we computed Eq. (3) for the Hubbard model using the cellular-dynamical mean-field theory ${ }^{36}$. In the (CDMFT)method,, 36 a cluster extending in a small number of sites is treated as the impurity and therefore the local (cluster) degrees of freedom are treated exactly. The rest of the lattice, the bath, is described by a multicomponent hybridization function.

All cluster-DMFT-based algorithms contain the following major components.

- An impurity solver, which evaluates the cluster Green function from the hybridization function.

- A self consistency condition which expresses the hybridization function with respect to the cluster Green function.

- A periodization procedure which connects the lattice quantities with the cluster quantities.

In the present application, we used a 4-site (plaquette) cluster. The coupling of the cluster to the bath is thus treated in a mean-field fashion and the cluster quantities are evaluated self consistently using the cumulant lattice reconstruction scheme ${ }^{37}$. Various impurity solvers have been proposed in the literature such as exact diagonalization and quantum Monte-Carlo. However those can only be implemented in imaginary time and an analytic continuation is required to obtain real time properties. A real time impurity solver is the non-crossing approximation (NCA), which is a first order perturbation theory with respect to the hybridization function. It has the advantage of being very fast and relatively easy to implement. The NCA has been a valuable tool for extracting the physics of the Anderson impurity models. The NCA Equations can be obtained by using the slave boson method 38 and they can be expressed with respect to the pseudo-particle resolvents and their self energies. We adopted this technique here in our implementation of the CDMFT method.

Using the CDMFT method, we obtained the oneparticle spectral function $A(\mathbf{k}, \omega)$ for various values of hole doping $\mathbf{x}$ and on-site Coulomb repulsion $\mathbf{U}$ (expressed in units of the nearest neighbor hopping integral t) . To calibrate the CDMFT method in this context, we first calculated the thermopower with a large value of $U$ for comparison purposes with the exact result in the atomic limit of Beni ${ }^{28}$. If this method is to be trusted, it should reproduce these exat results. The diamonds (green in color scale) in Fig. (44) represent the thermopower for $U=20 t$. The computed values match perfectly with the exact asymptotic atomic limit of Beni ${ }^{28}$, especially at large dopings. This striking agreement indicates two things. First, the method we use here provides an accurate quantitative description of the local physics behind the spectral weight shifts that leads to the thermopower in the atomic limit. Second, by $U=20 t$ the dynamical correction to the spectral weight is negligible. Consequently, for $U<20 t$, the sign change in the thermopower must occur at $x<1 / 3$. Indeed this is true. From Fig. (4), we see that both $U=8 t$ and $U=4 t$ at $T=0.1 t$ (slightly higher than the $290 \mathrm{~K}$ of the experiments) exhibit a sign change before the atomic limit value of $x=1 / 3$. For $U=4 t$, the sign change occurs at $x \approx 0.19$ whereas for $U=8 t, S$ vanishes at $x=0.21$ in agreement with the perturbative argument that as $t / U$ decreases, the critical value of the doping at which the thermopower changes sign must increase. For $U=8 t$, both the magnitude of the thermopower and the $S=0$ condition are in agreement with the experimental values in Fig. (1a). The inset shows the difference between the spectral weight above and below the chemical potential for $U=8 t$. As is evident, this quantity is finite even when the thermopower vanishes, in direct constrast to a non-interacting system in which particle-hole symmetry is essential for $S=0$. The deviation from the particlehole symmetric point, which lies in close proximity to the doping level at which $S$ vanishes, is due entirely to the momentum dependence of the spectral function at finite $t / U$. Consequently, the vanishing of the thermopower in the cuprates at a doping level significantly less than the atomic limit of $x=1 / 3$ is a signature that the dynamical contributions (the $t / U$ part) to the low-energy spectral weight and the momentum dependence of the spectral function cannot be ignored. Such an extreme sensitivity of the thermopower to the spectral weight redistribution obtains because the thermopower is generated by a thermal gradient, unlike an electrical gradient in a Hall measurement. That is, the thermopower is fundamentally an experiment about the conservation of particle number (as opposed to charge conservation in the Hall experiment), which is of course governed by electron spectral weight shifts. The final feature of this story

Based on the calculations presented here and our analysis of Eq. (21), we correlate the vanishing of the ther- 


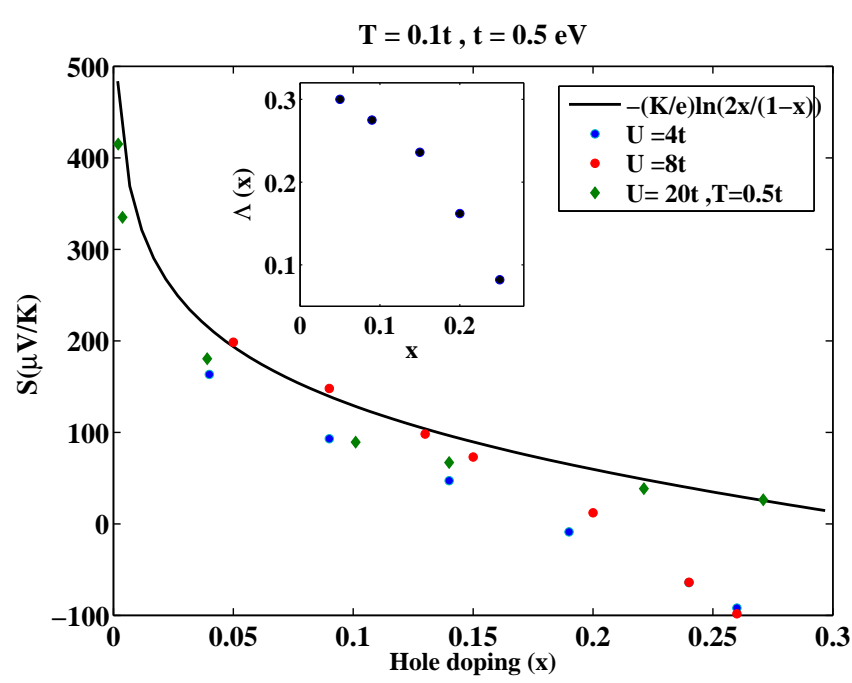

FIG. 4. Thermoelectric power computed for two different values of $U=8 t$, plotted as a function of hole doping, $x$. The solid line is Eq. (2), the exact result for the thermopower of a doped Mott insulator in the atomic limit. In the inset $\Lambda$ is the difference of the spectral weight above and below the chemical potential. As is evident, $S$ vanishes at a doping level slightly lower than that at which $\Lambda=0$. In a strongly correlated system, naive particle-hole symmetry is not essential for the vanishing of the thermopower.

mopower at the top of the dome for a wide range of cuprates to a proximity to the particle-hole symmetric state which is driven predominantly by mixing (dynamical spectral weight transfer) with states in the upper Hubbard band. As it is the dynamical part of $L$ that is relevant here, maximising $T_{c}$ is tied to states at high energy, in particular states that lie $2 \mathrm{eV}$ away from the chemical potential. This conclusion is consistent with the results from optical experiments $\underline{39,40}$ which indicate that the condensation energy in the superconducting state arises from electronic states that lie $2 \mathrm{eV}$ away from the chemical potential.

Aside from influencing the vanishing of the thermopower, dynamical spectral weight transfer also provides a general mechanism ${ }^{41}$ for the breakdown of Fermi liquid theory in doped Mott insulators in the absence of any symmetry breaking. In a Fermi liquid, the phase space available to add a particle at low energies (that is $L)$ is exhausted by enumerating the number of ways $\left(n_{h}\right)$ of adding an electron to the empty states. This principle follows from the Fermi liquid tenet that at any chemical potential, the number of quasiparticle excitations equals the number of bare electrons in the system. This ac- counting clearly breaks down in a real doped Mott system because the number of ways of adding an electron is simply $2 x$ but the phase space available to add a particle at low energies, namely $L$, exceeds ${ }^{32} 2 x$ as shown above. Hence, the number of low-energy particle addition states per electron per spin exceeds unity. As a result in a doped Mott insulator, there are electronic states at low energy that have no counterpart in the non-interacting system $\left(L / n_{h}>1\right)$ and Fermi liquid theory breaks down.

\section{FINAL REMARKS}

We have proposed here that the sign change of the thermopower in the cuprates is driven by dynamical spectral weight transfer. That our calculated values for the thermopower (see Fig. (4)) diamonds) agree perfectly with the exact atomic limit for $U=20 t$ lends credence to this interpretation. The cuprates reside at smaller values of $U=8 t$ where the dynamical mixing with the upper band is significant. Further, we propose here that close to the top of the dome where particle-hole symmetry is dynamically generated ${ }^{42.43}$, a quantum critical point obtains signalling a transition to weak-coupling physics. That is, in the strongly overdoped regime, the coupling to the highenergy scale ceases, giving rise to $L / n_{h}=1$, and traditional descriptions in terms of Landau quasiparticles apply. Recent soft x-ray Oxygen K-edge experiments 44 indicate that $L / n_{h}$ does saturate to a doping independent value in the overdoped regime once the pseudogap terminates as predicted here. Similar experiments should be performed as a function of temperature below and above the $T^{*}$ line. According to the theory we have recently constructed ${ }^{41}$ to explain the onset of non-Fermi liquid behaviour in a doped Mott insulator, $L / n_{h}$ below $T^{*}$ should increase. The increase should be given by $\alpha$, for which quantitative estimates have been obtained recently 34 . The fundamental problem of superconductivity in the cuprates reduces to uncloaking precisely how the high-energy scale physics mediated by dynamical spectral weight transfer creates a phase coherent condensate.

\section{ACKNOWLEDGMENTS}

This work was funded by the NSF DMR-0605769 and DMR-0940992, NSF PHY05-51164 (KITP) and Samsung scholarship (S. Hong). We also thank F. Krüger, T. Stanescu, R. Leigh, and G. Sawatzky for critical remarks.
1 T. Honma and P.H. Hor, Phys. Rev. B77, 184520 (2008).

${ }^{2}$ K. Matsuura et al., Phys. Rev. B 46, 11923 (1992).

3 S. D. Obertelli, J.R. Cooper and J.L.Tallon, Phys. Rev. B 46, 14928 (1992).
4 J. R. Cooper, B. Alavi, L. -W. Zhou, W. P. Beyermann, and G. Grüner, Phys. Rev. B 35 8794-8796 (1987).

5 R. C. yu, M. J. Naughton, X. Yan, P. M. Chaikin, F. Holtzberg, and R. L. Greene, Phys. Rev. B 37, 7963 (1988). 
6 J. Presland, J. L. Tallon, R. G. Buckley, R. S. Liu, and N. E. Flower, Physica C 17695 (1991).

7 T. Honma, private communication.

8 S. W. Moore et al., Phys. Rev. B 66, 060509 (2002).

9 S. L. Cooper, et al.,Phys. Rev. B 41, 11605 (1990).

10 S. Uchida et al., Phys. Rev. B 43, 7942 (1991).

11 D. van der Marel et al., Nature 425, 271 (2003).

12 S. W. Moore et al., Phys.Rev. B 46, 605091 (2002).

13 J. Bouvier et al., Phys. Rev. B 45, 8065 (1992).

14 Y. S. Lee et al.,Phys. Rev. 72, 54529 (2005).

15 M. Merz, et al., Phys. Rev. Lett. 80, 5192 (1998).

16 H. Kotegawa, et al., Phys. Rev. B 64, 064515 (2001).

17 A. Ino, et al., Phys. Rev. B 65, 094504 (2002).

18 T. Yoshida, et al., Phys. Rev. B 74, 224510 (2006).

19 T. Kondo, et al., Phys. Rev. B 72, 024533 (2005).

20 N. E. Hussey, M. Abdel-Jawad, A. Carrington, A. P. Mackenzie, and L. Balicas, Nature, 425, 814 (2003).

21 N. Nagaosa and P. A. Lee, Phys. Rev. Lett. 64, 2450 (1990).

22 G. C. McIntosh and A.B. Kaiser, Phys. Rev. B54, 12575 (1996).

${ }^{23}$ G. Hildebrand et al., Phys. Rev. B 56 , R4319 (1997).

${ }^{24}$ H. Eskes, L. H. Tjeng, and G. A. Sawatzky, Phys. Rev. B 41288 (1990).

25 M. B. J. Meinders, H. Eskes, and G. A. Sawatzky, Phys. Rev. Lett. 67, 1035 (1991).

26 C. M. Varma, S. Schmitt-Rink, E. Abrahams, Solid State Comm. 62, 681 (1987).

27 V. J. Emery, Phys. Rev. Lett. 582794 (1987).
28 G. Beni, Phys. Rev. B 10, 2186 (1974).

29 M. Jonson and G.D. Mahan, Phys. Rev. B 21, 4229 (1980).

30 B. S. Shastry, Phys. Rev. B 73, 085117/1-15 (2006).

31 M. B. J. Meinders, H. Eskes, and G. A. Sawatzky, Phys. Rev. B 48, 3916-3926 (1993).

32 A. B. Harris and R. V. Lange, Phys. Rev. 157, 295 (1967).

33 C. T. Chen, et al. Phys. Rev. Lett. 66, 104 (1991).

34 S. Chakraborty, S. Hong, and P. Phillips, Phys. Rev. B 81, 235135 (2010).

35 P. Kuiper, G. Kruizinga, J. Ghijsen, and G. A. Sawatzky Phys. Rev. Lett. 62 221-224 (1989).

${ }^{36}$ G. Kotliar, S. Y. Savrasov, G. Plsson and G. Biroli ,Phys. Rev. Lett. 87, 186401 (2001).

37 T. D. Stanescu and G. Kotliar, Phys. Rev. B 74, 125110 (2006).

38 P. Coleman, Phys. Rev. B 29, 3035 (1984).

39 H. J. A . Molegraaf, C. Presura, D. van der Marel, P. H. Kes, and M. Li, Science 295, 2239 (2002).

40 A. F. Santander-Syro, R. P. Lobo, N. Bontemps, Z. Konstantinovic, Z. Z. Li, and H. Raffy, Eur. Phys Lett. 62, 568 (2003).

41 P. Phillips, T. P. Choy, and R. G. Leigh, Phys. Rev. Lett. 99, 046404/1 (2007).

42 K. Haule and G. Kotliar, Phys. Rev. B 76, 092503 (2007).

43 N. S. Vidyadhiraja, A. Macridin, C. Sen, M. Jarrell, and M. Ma, Phys. Rev. Lett. 102, 206407 (2009).

44 D. C. Peets, et al., Phys. Rev. Lett. 103, 087402/1 (2009). 